\title{
Introduction to the Special Issue on Urban Computing and Smart Cities
}

In recent years, the urban networks infrastructure has undergone a fast expansion, which increasingly generates a large amount of data, such as human mobility data, human transactions data, regional weather and air quality data, and social connection data. These heterogeneous data sources convey rich information about a city and can enable intelligent solutions to solve various urban challenges, such as urban facility planning, air pollution, and so on. While on the one hand, these big urban data can help us to tackle big urban challenges, on the other hand, it is challenging to manage, analyze, and make sense of the big urban data.

The Urban Data Sciences special issue aims to publish work on multidisciplinary research across the areas of computer science, electrical engineering, environmental science, urban planning and development, social sciences, operation research, and industrial engineering on technologies, case studies, novel approaches, and visionary ideas related to data science solutions and data-driven applications to address real-world challenges for enabling smart cities. The objective of this special issue is to publish leading work in urban data science and present future challenges in this area. This special issue received 22 high-quality submissions, and 4 of them were accepted. The topics of the accepted articles are briefly introduced below.

In the article titled "Transfer Urban Human Mobility via POI Embedding over Multiple Cities," the authors proposed an embedding mechanism to fuse human mobility data and city POI data to improve the prediction performance with limited training data. Moreover, a deep learning architecture is proposed to combining CNN with LSTM to simultaneously capture both the spatiotemporal and geographical information from the enriched trajectories. The proposed method is evaluated with four citywide datasets.

The article titled "Empty Vehicle Redistribution with Time Windows in Autonomous Taxi Systems" addresses the topic of autonomous vehicle reservation strategies. The proposed approach is dynamic management of the vehicles using an Index-Based Redistribution Time Limited algorithm. The proposed algorithm improves existing algorithms by incorporating expected passenger arrivals and predicted waiting times limitations.

In the article titled "Scalable Belief Updating for Urban Air Quality Modeling and Prediction," the authors propose a scalable belief updating framework to predict future air quality and a nonparameter approach for statistical model learning. The proposed prediction model enables iterative updates for large-scale data. The authors analyzed the distribution of various pollutants and the influences of meteorology.

Moreover, in the last article of the special issue, entitled "WattScale: A Data-driven Approach for Energy Efficiency Analytics of Buildings at Scale," the authors presented a datadriven approach to identify the least energy-efficient buildings from a large population of buildings in a city or a region. The research topic has high practical value, as it can answer questions

\section{ACM Reference format:}

Y. Li, J. Bao, Z.-L. Zhang, and S. Benjaafar. 2021. Introduction to the Special Issue on Urban Computing and Smart Cities. ACM/IMS Trans. Data Sci. 2, 1, Article 2e (January 2021), 2 pages.

https://doi.org/10.1145/3441679

(C) 2021 Copyright held by the owner/author(s).

2577-3224/2021/01-ART2e

https://doi.org/10.1145/3441679

ACM/IMS Transactions on Data Science, Vol. 2, No. 1, Article 2e. Publication date: January 2021. 
for efficient house design for civil engineering, architecture design, and urban planning. The authors have implemented an open source tool with a user interface to perform analytics to generate reports for building efficiencies, which has great potential for real-world applications.

Yanhua Li Jie Bao

Zhi-Li Zhang

Saif Benjaafar

Guest Editors 